\title{
Expression of SPRR3 is associated with tumor cell proliferation and invasion in glioblastoma multiforme
}

\author{
QINGYANG LIU ${ }^{1}$, CHUANBAO ZHANG $^{2}$, GUOFO MA $^{2}$ and QUANGENG ZHANG ${ }^{1}$ \\ ${ }^{1}$ Department of Immunology, Institute of Basic Medical Sciences, Capital Medical University, Beijing 100069; \\ ${ }^{2}$ Department of Neurosurgery, Tiantan Hospital, Capital Medical University, Beijing 100050, P.R. China
}

Received May 10,2013; Accepted November 28, 2013

DOI: $10.3892 / 01.2013 .1736$

\begin{abstract}
Esophagin, also known as small proline-rich protein 3 (SPRR3), has been demonstrated to be important in the initiation and progression of numerous types of tumor, including colorectal and breast cancer. However, studies concerning the biological functions of SPRR3 in glioblastoma multiforme (GBM) are limited. Therefore, we aimed to identify the functions and molecular mechanisms underlying the role of SPRR3 in GBM. Hypomethylation of SPRR3 was observed and associated with a poor clinical outcome in GBM patients compared with healthy individuals by using gene methylation profiling. The present study was performed to investigate the expression status and effects of SPRR3 in GBM. The U251 cell line was used in the functional analyses. Cell growth was examined by MTT and colony formation assay. Cell invasion was measured using the Transwell invasion assay. The expression of SPRR3 in tissue samples was examined by immunohistochemistry. The results revealed that the overexpression of SPRR3 accelerates U251 cell proliferation and invasion. It was also observed that SPRR3 was markedly upregulated in $72.7 \%$ of GBM samples (24/33) compared with the normal tissue. These results suggest that an increased expression of SPRR3 is involved in tumorigenesis.
\end{abstract}

\section{Introduction}

Glioblastoma multiforme (GBM) is the most common primary brain tumor in adults and is associated with the highest rate of mortality (1). Numerous biological processes occur in tumorigenesis. $\mathrm{CpG}$ island hypermethylation silences tumor suppressor genes, whereas hypomethylation promotes the transcriptional activation of oncogenes and induces chromosomal

Correspondence to: Professor Quangeng Zhang, Department of Immunology, Institute of Basic Medical Sciences, Capital Medical University, 10 Xitoutiao, Youanmen, Fengtai, Beijing 100069, P.R. China

E-mail: quangengzhang@163.com

Key words: glioblastoma, SPRR3, cellular proliferation, invasion, methylation profiling instability $(2,3)$. Several aberrantly methylated genes have been reported in gliomas. For instance, the p53/Mdm2/p14ARF cell cycle control pathway genes are affected by $\mathrm{CpG}$ island promoter hypermethylation (4).

Gene methylation profiling is able to simultaneously evaluate thousands of individual gene methylation levels and reveal molecular signatures that reflect potential pathogenic mechanisms and are associated with survival. In the present study, we performed genome-wide gene methylation level analysis and identified SPRR3 as a candidate gene, which showed aberrant levels between GBM patients and healthy individuals.

The small proline-rich proteins (SPRRs) are encoded by a multigene family clustered within the epidermal differentiation complex on human chromosome 1q21 (5-7). SPRR proteins are known to be markers for terminal squamous cell differentiation; however, they also function in nonsquamous tissues (8). SPRR3 (also named esophagin) is abundantly expressed in oral and esophageal epithelia $(9,10)$. SPRR3 has been considered as a differentiation marker of squamous epithelium, since its expression is strictly correlated with keratinocyte terminal differentiation $(5,11)$. SPRR3 is frequently downregulated in esophageal squamous cell carcinoma (ESCC) and it has been demonstrated to suppress the tumorigenicity of ESCC cells $(4,7,12)$. However, it has previously been reported that SPRR3 is upregulated in colorectal and breast cancer $(13,14)$, suggesting that SPRR3 is associated with malignant tumorigenesis.

In the present study, we found that SPRR3 hypomethylation was associated with the clinical outcome in GBM patients. U251 cells demonstrated a significant decrease in proliferation and invasion following specific knockdown of SPRR3. Immunohistochemical staining demonstrated that SPRR3 was highly expressed in tumors compared with normal tissue.

\section{Materials and methods}

Patients, tissue samples and cell lines. All glioma samples included in the present study were obtained from the Chinese Glioma Genome Atlas (http://www.cgga.org.cn). The patients underwent surgical resection between January 2006 and December 2010, and subsequently received radiation therapy or concomitant and adjuvant temozolomide chemotherapy. Tumor tissue samples were obtained by surgical resection provided 
Table I. siRNA and negative control strand sequences.

Sense

Antisense

siRNA

Negative control
GCCAUAGUCUCUCUCUUAUTT

UUCUCCGAACGUGUCACGUTT
AUAAGAGAGAGACUAUGGCTT

ACGUGACACGUUCGGAGAATT

siRNA strand was from 5 ' to 3 '.

that the diagnosis of glioma was established according to the 2007 WHO classification, and eight normal brain tissues were included. The present study was approved by the institutional review boards of Beijing Tiantan Hospital (Beijing, China) and written informed consent was obtained from all patients. U251 glioma cells were purchased from the Chinese Academy of Sciences Cell Bank (Kunming, Yunnan, China). U251 cells were cultured in Dulbecco's modified Eagle's medium (DMEM) supplemented with 10\% fetal bovine serum (FBS). All cells were maintained in a $37^{\circ} \mathrm{C}, 5 \% \mathrm{CO}_{2}$ incubator and routinely passaged at 2-3 day intervals.

Genomic DNA extraction and DNA methylation profiling. All the tissue samples were immediately snap-frozen in liquid nitrogen following surgery. Genomic DNA from frozen tumor tissues was extracted using the QIAamp DNA Mini kit (Qiagen, Hilden, Germany) according to the manufacturer's instructions. DNA concentrations were measured using a NanoDrop ND-1000 spectrophotometer (NanoDrop Technologies, Houston, TX, USA). Illumina Infinium HumanMethylation27 BeadChips (Illumina Inc., San Diego, CA, USA) were used as previously described (15). Methylation of 27,578 $\mathrm{CpG}$ sites at 14,475 consensus coding sequencing sites was performed following the manufacturer's instructions at the Wellcome Trust Centre for the Human Genetics Genomics Lab (Oxford, UK). The array results were analyzed with the BeadStudio software (Illumina Inc.).

SPRR3 gene knockdown by siRNA. Logarithmically growing cells were seeded at a density of $10^{5}$ cells per $6-\mathrm{cm}$ dish. Oligonucleotide transfection was performed using the siRNA and siRNA negative control (NC; Table I) that were chemically synthesized by the Shanghai GenePharma Company (Shanghai, China). Cells were transfected using Lipofectamine 2000 reagent (Invitrogen Life Technologies, Carlsbad, CA, USA). Transfection complexes were prepared according to the manufacturer's instructions and added directly to the glioma cells, resulting in a final oligonucleotide concentration of $10 \mathrm{nmol} / \mathrm{l}$. The transfection medium was replaced $6 \mathrm{~h}$ post-transfection. Cells were used for in vitro functional assay.

Western blot analysis. Following cell treatment, in order to determine the levels of SPRR3 expression, total protein was isolated in lysis buffer. Equal amounts of protein $(15 \mu \mathrm{g})$ were loaded into the sample wells and separated on a $10 \%$ SDS-polyacrylamide gel, and transferred onto polyvinylidene difluoride membranes. Immunoblot analysis was performed with the mouse antibodies against SPRR3 (ab58233; Abcam, Hong Kong, China; 1:1,000 dilution). $\beta$-actin (anti- $\beta$-actin antibody was obtained from Proteintech Group, Chicago, IL, USA; 1:4,000 dilution) was reblotted to check for equal loading of the gel.

MTT and colony formation assay. The MTT assay was used to determine relative cell growth. U251 cells were plated at a density of 5,000 cells per well $24 \mathrm{~h}$ after transfection with siRNA in 96-well plates with six replicate wells for each condition. For quantitation of cell viability, cultures were stained after 5 days. A cell growth assay was performed using MTT (thiazolyl blue). In brief, $20 \mu 1$ of $5 \mathrm{mg} / \mathrm{ml}$ MTT solution was added to each well and incubated for $4 \mathrm{~h}$ at $37^{\circ} \mathrm{C}$. The cell viability was determined at an absorbance of $490 \mathrm{~nm}$ following solubilization in $150 \mu \mathrm{l}$ DMSO. All data points represent the mean of a minimum of six wells. A colony formation assay was performed and briefly U251 cells were transfected with siRNA or the NC for $48 \mathrm{~h}$, and seeded into the individual wells of a six-orifice plate $(2,000$ per orifice). Following culture for 14 days, all orifices were washed with PBS and stained with crystal violet. The number of colonies with $>30$ cells was counted. The colonies were manually counted using a microscope (Leica DM6000 B; Upright Microscopes, Wetzlar, Germany).

Transwell invasion assay. Cell culture chambers (24-well) with Transwell inserts (Corning Life Sciences, Corning, NY, USA) with an $8-\mu \mathrm{m}$ pore membrane precoated with Matrigel (BD Biosciences, San Jose, CA, USA) were used in the Transwell invasion assay. U251 cells were plated at a density of $1 \times 10^{4}$ per upper well in $200 \mu 1$ culture medium (DMEM, without FBS) and the lower chamber was filled with $500 \mu \mathrm{l}$ of medium (DMEM, 10\% FBS) in the set control group, siRNA control group and siRNA group. The cells were incubated at $37^{\circ} \mathrm{C}$ and allowed to invade for $24 \mathrm{~h}$, following which, the upper surface of the membrane of the non-invading cells was removed by scrubbing with a cotton-tipped swab. Cells on the lower surface of the filter were fixed for $20 \mathrm{~min}$ in absolute ethyl alcohol and stained with crystal violet after being air-dried briefly. The mean number of invaded cells was counted from five preselected microscopic fields at magnification x200 and all experiments were performed in triplicate.

Immunohistochemistry. Surgical specimens were fixed in formalin, routinely processed and paraffin embedded, then cut into 4- $\mu \mathrm{m}$ sections, deparaffinized with xylene and rehydrated. Sections were submerged in EDTA ( $\mathrm{pH} 8.0$ ), autoclaved for antigen retrieval and then treated with $3 \%$ hydrogen peroxide, followed by incubation with $1 \%$ FBS. Anti-SPRR3 antibody (Abcam; mouse monoclonal; 1:200 dilution) was added as a primary antibody and incubated at $4^{\circ} \mathrm{C}$ for $2 \mathrm{~h}$. Normal mouse 
Table II. Variables associated with the methylation level of SPRR3 in 42 glioma samples.

\begin{tabular}{lcccc}
\hline & & & SPRR3 methylation level \\
\cline { 3 - 5 } Variable & No. of patients & Median OS (days) & Low & High \\
\hline Gender & & & & 22 \\
Male & 26 & 261 & 4 & 13 \\
Female & 16 & 241 & 3 & 21 \\
Age (years) & 28 & & & 0.617 \\
$\leq 50$ & 14 & 348 & 0 & 14 \\
$>50$ & & 211 & 0 & \\
\hline
\end{tabular}

OS, overall survival; SPRR3, small proline-rich protein 3.

A

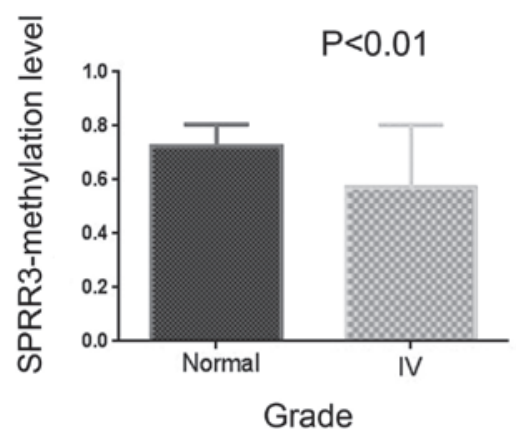

B

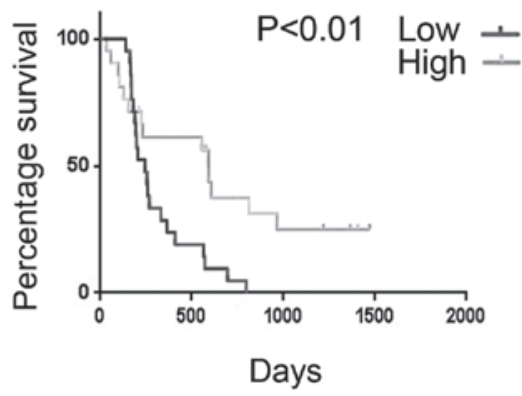

Figure 1. Methylation level of SPRR3 in gliomas and association with survival in glioblastoma multiforme. (A) Methylation levels of SPRR3 in healthy individuals and high-grade gliomas, analyzed by DNA methylation profiling containing 227 frozen glioma tissues. The methylation level of SPRR3 was significantly lower in high-grage glioma patients compared with those in healthy individuals $(\mathrm{P}<0.001)$. (B) Kaplan-Meier survival curves according to the methylation level of SPRR3 in 42 frozen high-grade glioma tissues. The log-rank test was used to calculate P-values. SPRR3, small proline-rich protein 3.

serum was used for the NC and horseradish peroxidase-labeled secondary antibody (Santa Cruz Biotechnology, Inc., Santa Cruz, CA, USA) was applied and incubated for $45 \mathrm{~min}$ at $37^{\circ} \mathrm{C}$, followed by $5 \mathrm{~min}$ incubation at room temperature with DAB for color development. Finally, the sections were counterstained with hematoxylin and mounted with Permount (BIOS, Beijing, China). The immunohistochemical staining results were visualized and images were captured under a bright-field microscope (Olympus BX-51; Olympus Optical Co., Ltd., Tokyo, Japan). The SPRR3 cytoplasmic expression was classified into two categories determined by combining the proportion of positively stained tumor cells and the intensity of staining. The intensity of staining was scored by two investigators without knowledge of clinical information on a scale of 0 to 3 ( 0 , negative; 1 , slightly positive; 2 , moderately positive; 3 , intensely positive). A score of 0 and 1 or 2 and 3 indicated low or high expression, respectively.

Statistical and bioinformatics analysis. Kaplan-Meier survival analysis was used to estimate the survival distributions and the overall survival (OS) time was calculated from the date of diagnosis until mortality or the last follow-up contact. Significant differences among the groups were determined using Student's t-test. All analyses were two-tailed. $\mathrm{P}<0.05$ was considered to indicate a statistically significant difference. Analyses were performed using Matlab 2009b (MathWorks, Natick, MA, USA), GraphPad Prism (GraphPad Software Inc., La Jolla, CA, USA) and SPSS version 13.0 (SPSS, Inc., Chicago, IL, USA).

\section{Results}

DNA methylation profile analysis. To investigate the molecular changes associated with GBM tumorigenesis, we analyzed the gene methylation level in 42 patients with GBM and eight healthy individuals using a DNA methylation profile (Table II). The methylation levels of 14,475 genes were analyzed using t-tests. Among these genes, SPRR3 was demonstrated to be associated with molecular changes and its methylation level was significantly lower in GBM patients compared with healthy individuals (Fig. 1). The OS time was measured through Kaplan-Meier survival curve analysis and the results revealed that glioma patients with a low methylation level of SPRR3 had significantly lower progression-free survival rates $(\mathrm{P}=0.010)$ and $\mathrm{OS}$ times $(\mathrm{P}=0.007)$ than patients with a high methylation level. Kaplan-Meier survival curves, according to the methylation level of SPRR3 in 42 frozen glioma tissues, demonstrated that SPRR3 hypomethylation was associated with a poor clinical outcome in GBM patients (Fig. 1). These results indicated that the methylation level of SPRR3 is an independent prognostic marker in glioma patients. 
A

B

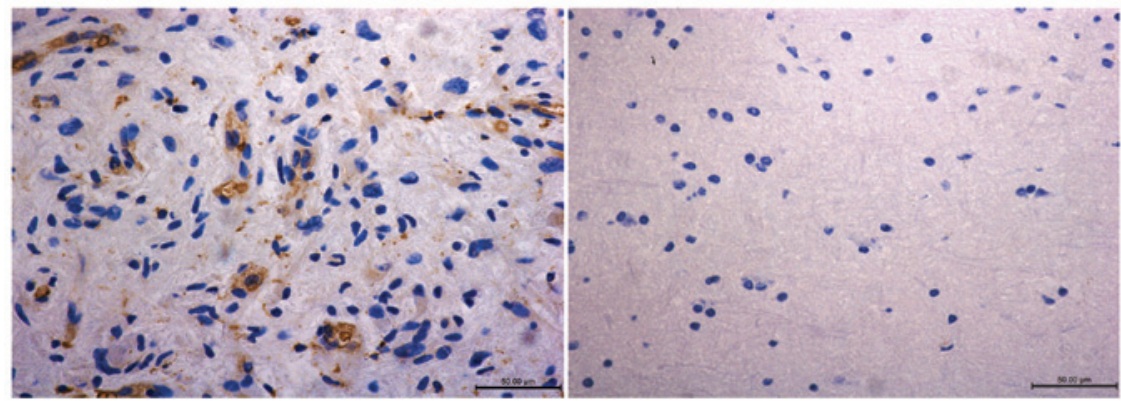

Figure 2. SPRR3 overexpression in glioma. Immunohistochemical staining demonstrated that SPRR3 was highly expressed in glioblastoma multiforme compared with the normal tissue (magnification, x400). (A) Glioblastoma (WHO grade IV); (B) normal tissue of the brain. SPRR3 was expressed in stroma. SPRR3, small proline-rich protein 3.

A

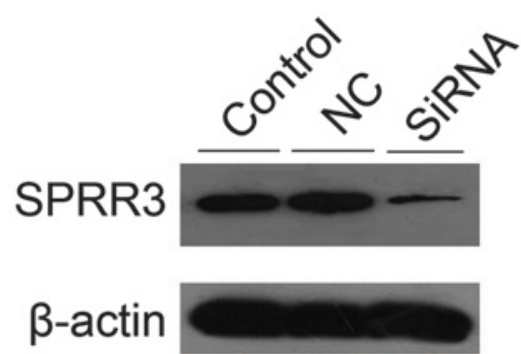

C

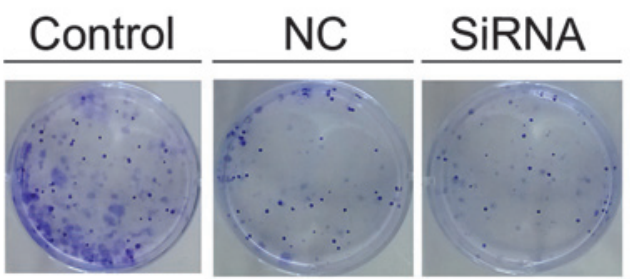

D

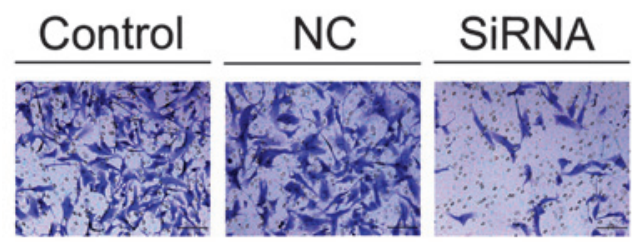

B
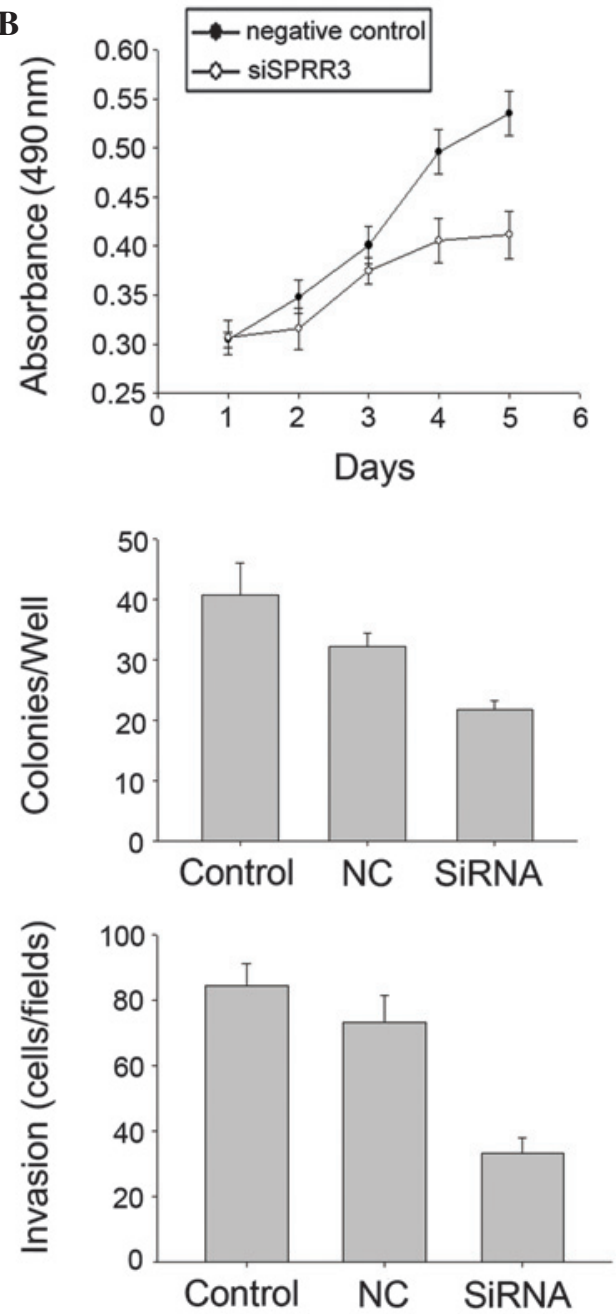

Figure 3. SPRR3 promotes the proliferation and invasion of U251 cell lines. (A) Expression of SPRR3 was significantly reduced following knockdown by siRNA. $\beta$-actin was used as the control. (B) Proliferation of U251 cell lines was assessed by MTT assay and a decrease in absorbance was observed following the knockdown of SPRR3. (C) Number of colonies formed by cells treated with siRNA of SPRR3 was decreased compared with the control cells (P<0.05). (D) Invasion of the U251 cell line assessed by Transwell assay (magnification, x200). The number of invading cells was significantly less in the siRNA group compared with the control and siRNA control groups $(\mathrm{P}<0.05)$. NC, negative control; SPRR3, small proline-rich protein 3.

SPRR3 is upregulated in glioblastoma. Samples of the tumor immunohistochemical staining demonstrated that SPRR3 was highly expressed in tumors compared with the normal tissue (Fig. 2). The quantification of immunohistochemical signals for 33 GBM samples revealed that 24 of the samples demonstrated positive staining for SPRR3 (72.7\%) while only $11.1 \%$ of the normal tissue stained positively for SPRR 3 (one in nine samples). No significant differences in patient age and gender were identified (Table III). We also measured the OS time according to SPRR3 expression levels in glioma patients 
Table III. Variables associated with the expression of SPRR3 in 34 glioma samples.

\begin{tabular}{|c|c|c|c|c|c|}
\hline \multirow[b]{2}{*}{ Variable } & \multirow[b]{2}{*}{ No. of patients } & \multirow[b]{2}{*}{ Median OS (days) } & \multicolumn{2}{|c|}{ SPRR3 expression level } & \multirow[b]{2}{*}{ P-value } \\
\hline & & & Low & High & \\
\hline Gender & & & & & 0.935 \\
\hline Male & 23 & 447 & 5 & 18 & \\
\hline Female & 11 & 315 & 2 & 9 & \\
\hline Age (years) & & & & & 0.798 \\
\hline$\leq 50$ & 14 & 532.5 & 2 & 12 & \\
\hline$>50$ & 20 & 315 & 5 & 15 & \\
\hline
\end{tabular}

OS, overall survival; SPRR3, small proline-rich protein 3.

through Kaplan-Meier survival estimates; however, the results revealed that there was no significant correlation between the two subgroups with high or low SPRR3 expression (high expression, $\geq 2$; low expression, $<2$ ).

Identification of SPRR3-associated proliferation and invasion signature in GBM. As the results of the immunohistochemical staining demonstrated that SPRR3 was highly expressed in GBM samples, we investigated the effect of SPRR3 on the proliferation and invasion of GBM cells. SPRR3 siRNA was used to specifically knock down SPRR3 expression in U251 cell lines (Fig. 3). The MTT assay demonstrated a significant decrease in proliferation in siRNA U251 cell lines compared with the cells transfected with the control (Fig. 3). The same result was found in the colony formation assay and the siRNA U251 cell lines demonstrated a decrease in focus numbers compared with the control ( $\mathrm{P}<0.05$; Fig. 3). Cell invasion was measured using the Transwell invasion assay and the results demonstrated that U251 cell lines significantly attenuated invasiveness following SPRR3 knockdown, as indicated by a marked decrease in the number of invaded cells $(\mathrm{P}<0.05$; Fig. 3$)$.

\section{Discussion}

SPRR3 promoted GBM cell (U251) proliferation and invasion. The results of the immunohistochemical staining demonstrated that SPRR3 was upregulated in the tissue samples of more than half of the GBM patients (72.7\%) and the methylation level of SPRR3 was correlated with the clinical outcome in glioma patients validated by DNA methylation profile analysis. The present study, to the best of our knowledge, is the first to examine the functions and methylation level of SPRR3 in GBM.

GBM is the most common and aggressive primary brain tumor in adults. Its prognosis remains extremely poor, despite multimodal treatment by surgery, radiotherapy and chemotherapy (16). Aberrations in DNA methylation patterns may have critical effects on tumor initiation and progression (17). DNA hypermethylation of specific genes and DNA hypomethylation commonly affecting repetitive DNA are observed in brain cancers (18-24).

In order to investigate the potential molecular mechanisms underlying GBM tumorigenesis, we analyzed gene methylation levels in 227 patients with glioma (141 low-grade gliomas,
44 anaplastic gliomas and 42 GBMs) and eight healthy individuals by using a DNA methylation profile. We found that the methylation levels of several genes were significantly altered (SPRR3, TES, RGN, FAM12B, AJAP1, PDE4C and SPRR2D). Among these genes, we found that SPRR3 hypomethylation was a common event in the majority of cases, regardless of the sex and age of the patients, and SPRR3 hypomethylation was also demonstrated to be associated with an adverse prognosis. In the present study, we found that SPRR3 was hypomethylated in GBM. Previously, Ammerpohl et al (25) demonstrated the same result in cirrhotic liver and hepatocellular carcinoma. Mayol et al also found that SPRR3 hypomethylation affected cancer-related biological functions and genes relevant to neuroblastoma pathogenesis (26).

SPRR3 is frequently downregulated in ESCC, where it is known to inhibit tumorigenesis. Notably, in contrast to the findings in ESCC, in the present study, SPRR3 promoted GBM cell (U251) proliferation and invasion, and samples from the tumor immunohistochemical staining demonstrated that SPRR3 was highly expressed in the majority of tumors compared with the normal tissue. Previously, SPRR3 was demonstrated to be upregulated in colorectal and breast cancer, and these results were in accordance with our findings. Our study suggests that upregulation of SPRR3 occurs in GBM tumorigenesis.

Considering the vital role of cellular proliferation and invasion in GBM pathogenesis, we investigated the effect of the overexpression of SPRR3 on GBM cell lines. SPRR3 siRNA was used to specifically knock down SPRR3 expression. The MTT assay demonstrated a significant decrease in proliferation and was consistent with the colony formation assay results in the U251 glioma cell line following transfection (Fig. 3). Cellular proliferation is one of the most important biological processes in tumorigenesis due to its role in growth and in the maintenance of tissue homeostasis $(27,28)$. Each type of human cancer often has specific signaling pathways to rely on for cellular proliferation (29). In colorectal cancer, in which SPRR3 was upregulated and accelerated cell proliferation, it was proposed that the effect of SPRR3 in promoting colorectal tumorigenesis is associated with the degradation of p53 caused by AKT activation (13). The same results were also found in breast cancer. SPRR3 has been demonstrated to promote breast cancer cell proliferation by enhancing p53 degradation via the AKT and MAPK pathways (14). We used a similar 
experiment and demonstrated the same results in the U251 glioma cell line. AKT signaling has been implicated in angiogenesis, the promotion of tumor invasion as well as in tumor cell survival $(30,31)$. In particular, a higher proliferation index of the tumor cells has been associated with shorter survival rates $(32,33)$. As AKT signaling promotes tumor invasion, we used a Transwell invasion assay to measure cell invasiveness and the results demonstrated that the invasiveness of U251 cell lines significantly decreased following the knock down of SPRR3 expression.

The present study demonstrated that SPRR3 is hypomethylated and upregulated in glioblastoma, and the integrated microarray analysis revealed that SPRR3 hypomethylation was associated with an adverse prognosis. We also demonstrated that the overexpression of SPRR3 may be associated with cell proliferation and invasion in GBM, and that it may be a candidate biomarker for GBM.

\section{Acknowledgements}

We would like to thank Professor Zhang (Capital Medical University, Beijing, China) for the technical support and Beijing Tiantan Hospital (Beijing, China). This study was supported by grants from the National High Technology Research and Development Program (no. 2012AA02A508) and the International Science and Technology Cooperation Program (no. 2012DFA30470).

\section{References}

1. Parsons DW, Jones S, Zhang X, et al: An integrated genomic analysis of human glioblastoma multiforme. Science 321: 1807-1812, 2008.

2. Herman JG and Baylin SB: Gene silencing in cancer in association with promoter hypermethylation. N Engl J Med 349: 2042-2054, 2003.

3. Karpf AR and Matsui S: Genetic disruption of cytosine DNA methyltransferase enzymes induces chromosomal instability in human cancer cells. Cancer Res 65: 8635-8639, 2005.

4. Bello MJ and Rey JA: The p53/Mdm2/p14ARF cell cycle control pathway genes may be inactivated by genetic and epigenetic mechanisms in gliomas. Cancer Genet Cytogenet 164: 172-173, 2006.

5. Gibbs S, Fijneman R, Wiegant J, van Kessel AG, van De Putte P and Backendorf $\mathrm{C}$ : Molecular characterization and evolution of the SPRR family of keratinocyte differentiation markers encoding small proline-rich proteins. Genomics 16: 630-637, 1993.

6. Steinert PM, Candi E, Kartasova T and Marekov L: Small proline-rich proteins are cross-bridging proteins in the cornified cell envelopes of stratified squamous epithelia. J Struct Biol 122: 76-85, 1998.

7. Zhang Y, Feng YB, Shen XM, et al: Exogenous expression of Esophagin/SPRR3 attenuates the tumorigenicity of esophageal squamous cell carcinoma cells via promoting apoptosis. Int J Cancer 122: 260-266, 2008.

8. Tesfaigzi J, Th'ng J, Hotchkiss JA, Harkema JR and Wright PS: A small proline-rich protein, SPRR1, is upregulated early during tobacco smoke-induced squamous metaplasia in rat nasal epithelia. Am J Respir Cell Mol Biol 14: 478-486, 1996.

9. Fischer DF, Sark MW, Lehtola MM, Gibbs S, van de Putte P and Backendorf C: Structure and evolution of the human SPRR3 gene: implications for function and regulation. Genomics 55: 88-99, 1999.

10. Cabral A, Sayin A, de Winter S, Fischer DF, Pavel S and Backendorf C: SPRR4, a novel cornified envelope precursor: UV-dependent epidermal expression and selective incorporation into fragile envelopes. J Cell Sci 114: 3837-3843, 2001.
11. Abraham JM, Wang S, Suzuki H, et al: Esophagin cDNA cloning and characterization: a tissue-specific member of the small proline-rich protein family that is not expressed in esophageal tumors. Cell Growth Differ 7: 855-860, 1996.

12. Chen BS, Wang MR, Cai Y, et al: Decreased expression of SPRR3 in Chinese human oesophageal cancer. Carcinogenesis 21: 2147-2150, 2000

13. Cho DH, Jo YK, Roh SA, et al: Upregulation of SPRR3 promotes colorectal tumorigenesis. Mol Med 16: 271-277, 2010.

14. Kim JC, Yu JH, Cho YK, et al: Expression of SPRR3 is associated with tumor cell proliferation in less advanced stages of breast cancer. Breast Cancer Res Treat 133: 909-916, 2012.

15. Hill VK, Ricketts C, Bieche I, et al: Genome-wide DNA methylation profiling of $\mathrm{CpG}$ islands in breast cancer identifies novel genes associated with tumorigenicity. Cancer Res 71: 2988-2999, 2011.

16. Wen PY and Kesari S: Malignant gliomas in adults. N Engl J Med 359: 492-507, 2008.

17. Nagarajan RP and Costello JF: Epigenetic mechanisms in glioblastoma multiforme. Semin Cancer Biol 19: 188-197, 2009.

18. Fanelli M, Caprodossi S, Ricci-Vitiani L, et al: Loss of pericentromeric DNA methylation pattern in human glioblastoma is associated with altered DNA methyltransferases expression and involves the stem cell compartment. Oncogene 27: 358-365, 2008.

19. Kim TY, Zhong S, Fields CR, Kim JH and Robertson KD: Epigenomic profiling reveals novel and frequent targets of aberrant DNA methylation-mediated silencing in malignant glioma. Cancer Res 66: 7490-7501, 2006.

20. Foltz G, Ryu GY, Yoon JG, et al: Genome-wide analysis of epigenetic silencing identifies BEX1 and BEX2 as candidate tumor suppressor genes in malignant glioma. Cancer Res 66: 6665-6674, 2006.

21. Cadieux B, Ching TT, VandenBerg SR and Costello JF: Genome-wide hypomethylation in human glioblastomas associated with specific copy number alteration, methylenetetrahydrofolate reductase allele status, and increased proliferation. Cancer Res 66: 8469-8476, 2006.

22. Yu J, Zhang $\mathrm{H}, \mathrm{Gu} \mathrm{J}$, et al: Methylation profiles of thirty four promoter- $\mathrm{CpG}$ islands and concordant methylation behaviours of sixteen genes that may contribute to carcinogenesis of astrocytoma. BMC Cancer 14: 65, 2004.

23. Uhlmann K, Rohde K, Zeller C, et al: Distinct methylation profiles of glioma subtypes. Int J Cancer 106: 52-59, 2003.

24. Costello JF, Frühwald MC, Smiraglia DJ, et al: Aberrant CpG-island methylationhasnon-randomand tumour-type-specific patterns. Nat Genet 24: 132-138, 2000.

25. Ammerpohl O, Pratschke J, Schafmayer C, et al: Distinct DNA methylation patterns in cirrhotic liver and hepatocellular carcinoma. Int J Cancer 130: 1319-1328, 2012.

26. Mayol G, Martín-Subero JI, Ríos J, et al: DNA hypomethylation affects cancer-related biological functions and genes relevant in neuroblastoma pathogenesis. PLoS One 7: e48401, 2012.

27. Fritz V and Fajas L: Metabolism and proliferation share common regulatory pathways in cancer cells. Oncogene 29: 4369-4377, 2010.

28. Davis CD, Emenaker NJ and Milner JA: Cellular proliferation, apoptosis and angiogenesis: molecular targets for nutritional preemption of cancer. Semin Oncol 37: 243-257, 2010.

29. Weinstein IB and Joe A: Oncogene addiction. Cancer Res 68: 3077-3080, 2008.

30. McCubrey JA, Steelman LS, Abrams SL, et al: Roles of the RAF/MEK/ERK and PI3K/PTEN/AKT pathways in malignant transformation and drug resistance. Adv Enzyme Regul 46: 249-279, 2006.

31. Testa JR and Bellacosa A: AKT plays a central role in tumorigenesis. Proc Natl Acad Sci USA 98: 10983-10985, 2001.

32. Protzel C, Knoedel J, Zimmermann U, Woenckhaus C, Poetsch $\mathrm{M}$ and Giebel $\mathrm{J}$ : Expression of proliferation marker Ki67 correlates to occurrence of metastasis and prognosis, histological subtypes and HPV DNA detection in penile carcinomas. Histol Histopathol 22: 1197-1204, 2007.

33. Yamashita Y, Kasugai I, Sato M, et al: CDC25A mRNA levels significantly correlate with Ki-67 expression in human glioma samples. J Neurooncol 100: 43-49, 2010. 